Discrete Comput Geom 31:587-611 (2004)

DOI: $10.1007 / \mathrm{s} 00454-004-2851-2$

\title{
Properties of $\mathcal{R}$-Sausages
}

\author{
Badri Toppur ${ }^{1}$ and J. MacGregor Smith ${ }^{2}$ \\ ${ }^{1}$ T. A. Pai Management Institute, \\ Manipal, Karnataka 576119, India \\ tbadri@mail.tapmi.org \\ ${ }^{2}$ Department of MIE, University of Massachusetts, \\ 160 Governor's Drive, Amherst, MA 01003, USA \\ jmsmith@ecs.umass.edu
}

\begin{abstract}
This paper is concerned with the Steiner ratio. A number of properties about the structure of the flat sausage and $\mathcal{R}$-Sausage convex polytopes yielding the best Steiner ratio in two- and three-dimensional Euclidean space, and the topology of the Steiner Minimal Tree for the corresponding vertex sets, are presented.
\end{abstract}

\section{Outline}

The most recent survey of the Steiner problem has been conducted by Cieslik. In his work The Steiner ratio he recounts the history of the problem [1]. Cieslik clarifies that the problem is attributed to Steiner by Courant and Robbins: "A very simple but instructive problem was treated by Jacob Steiner, the famous representative of geometry at the University of Berlin in the early nineteenth century. Three villages A, B, C are to be joined by a system of roads of minimum total length" [2]. The authentic attribution is to Fermat who, in the early 17 th century, stated the problem in the following form: given three points, find a fourth point that minimizes the sum of the distances from each of the three points to the fourth. We call this Fermat's problem or the Steiner problem, depending on whether the set consists of three points or more than three points, respectively.

Definition 1. Consider a set of $N$ vertices $V=\left(v_{1}, v_{2}, \ldots, v_{N}\right)$ with Cartesian coordinates in Euclidean space $E^{d}$. The Steiner Minimal Tree (SMT) problem is to construct a tree $T$ spanning $V$ with possible candidate vertices from another set $S$ (distinct from $V$ ) so as to minimize the overall interconnecting length of $T$. 
To situate the Steiner problem in the literature related to sciences and engineering, one must also take bearings with respect to the canonical works of Garey and Johnson, and their definitions of computational and algorithmic complexity. The Euclidean SMT (ESMT) problem is not in the class NP [9]. It has also been shown to be NP-hard in the plane [8]. Unless $\mathrm{P}=\mathrm{NP}$, which seems an undecidable proposition, Garey and Johnson also showed that no polynomial approximation scheme can exist for either the ESMT or the Rectilinear SMT problem. This they did by creating a discrete version of the ESMT problem which can then be shown to be NP-Complete, by reducing it in polynomial time to the "exact cover by three sets" problem which is known to be NP-Complete. Thus the ESMT problem is at least as hard as the discrete ESMT problem and is therefore as hard to solve, if not harder, as any NP-Complete problem, e.g., the Traveling Salesman Problem. One is therefore forced to consider algorithms that take exponential time or use other constant time approximation schemes. An exact algorithm for optimizing $d$ dimensional, full Steiner topologies was given by Smith [14]. Gilbert and Pollak showed that optimizing a pre-specified Steiner topology is a strictly convex optimization problem [10]. However, even when the topology is specified, numerical optimization techniques encounter difficulties. Both data-set size and computational time have to be taken into account as criteria in evaluating the efficiency of any algorithm.

Some background information on the Steiner ratio is provided in Section 2. The progressive construction of the flat sausage is presented in Section 3. The path topology, an important full Steiner tree topology, is defined. We also introduce a representation of the Steiner trees for regular triangulated complexes as permutations in a finite alphabet. Each of the letters is a characterization of a Steiner point in terms of its neighboring points. In Section 4, structural properties of the flat sausage are proven after providing substantial experimental evidence and formulating conjectures. Computational evidence that the flat sausage has the path topology as the optimal SMT topology is presented. This is followed by a non-inductive proof that this is so for a flat sausage: the minimizing direction of the objective function, the length of the SMT is towards the centroid of the triangle, in the case of an equilateral triangle. In the case when two flat sausage elements are combined by conjoining their outermost edge, a similar, though not equilateral, triangle is the focus of attention. After a split operation at this common vertex, the locus of the resulting Steiner point is directly towards the axis of the composite flat sausage, and does not disrupt the topology of the element at parts which are close to this triangle. This process can therefore be repeated ad infinitum to generate the entire semi-group, including the infinite flat sausage. Properties of the ribbon sausage or $\mathcal{R}$-sausage are proven in Section 5, after providing substantial experimental evidence and formulating conjectures. The calculus of variations technique used to prove that the path topology is optimal for flat sausages, is then shown to be valid for the $\mathcal{R}$-sausage too. The last section provides a summary and conclusion.

\section{The Steiner Ratio}

The optimal Steiner ratio is a measure of the improvement of one interconnecting network over another interconnecting network:

$$
\rho_{d}=\inf _{V \in E^{d}} \frac{\text { Total length of ESMT }(V)}{\text { Total length of EMST }(V)} .
$$


In the above formula, ESMT is an acronym for Euclidean Steiner Minimal Tree, and EMST stands for Euclidean Minimum Spanning Tree. Gilbert and Pollack conjectured that this ratio was achieved by the regular simplex in each dimension.

\subsection{The Steiner Ratio in Two and Higher Dimensions}

It was shown by Du and Hwang, confirming the conjecture of Gilbert and Pollak, that $\rho_{2}=\sqrt{3} / 2 \approx 0.866$ in two dimensions [5]. This is achieved when the vertices are the extreme points of an equilateral triangle. Du and Smith were also able to prove that the higher-order simplexes were not the optimal point configurations [6]. Although more than one exact algorithm exists for computing Minimum Spanning Trees (MSTs), geometric algorithms for the SMT problem in dimensions greater than two have proven extremely difficult. In three dimensions the equilateral tetrahedron is optimal among all possible four point configurations. While the singleton simplex failed to realize the optimal configuration for $E^{3}$, it eventually leads to the search for the optimal configuration by looking at two tetrahedra as well as by gluing various types and combinations of Platonic solids (cubes, tetrahedra, octahedra, etc.) together. Surprisingly, a breakthrough came when three tetrahedra were configured, where $\rho_{3}$ dramatically dropped and continued to drop for increasing numbers of tetrahedra. Thus, the Steiner ratio turns out to be asymptotically achieved for an infinite object rather than for a fixed symmetric and geometric polyhedron as was expected [13].

\subsection{Definition of the R-Sausage}

The $d$-dimensional point set which Smith and MacGregor Smith call the $R$-sausage may be generated in the following way [13]:

Definition 2. Start with a unit diameter ball in d-space. Successively add unit balls so that the $N$ th ball added is always touching the $\min (d, N-1)$ most recently added balls.

This procedure uniquely defines an infinite sequence of interior-disjoint numbered $d$-balls. The centers of these balls form a countably infinite discrete point set, which is called the $R$-sausage. A point set consisting of $N$ points of the $d$-sausage is called the $N$ point $R$-sausage. The 1-sausage is simply the integers on the real axis. The 2sausage is an infinite version of a lattice of equilateral triangles which we refer to as the "flat sausage" for convenience. The Steiner ratio of this 2-sausage converges to the optimal Steiner ratio in the two dimensions, the same as the simplex. This evidence, albeit computational, gives credence to the supposition that the higher-dimensional $R$ sausages yield the optimal Steiner ratios in their respective dimensions. A path of tree topologies has been suggested, which may top the $R$-sausage with respect to the Steiner ratio in dimensions greater than or equal to 80. Coxeter, in 1961, in his textbook on geometry, showed that 28 tetrahedra conjoined, as an $R$-sausage was aperiodic, about an axis through the centroids of the tetrahedra. However, he makes no reference to Steiner trees in his book [3]. Smith and MacGregor Smith performed an extensive study of the Steiner ratio [13]. Du and Smith computed upper bounds $g(d)$ on $\rho_{d}$ for $d$-sausages. 
$d$-Sausages are the $d$-dimensional counterparts of the flat sausage and the $R$-sausage [6]. The best rigorous lower bound on $\rho_{3}$ is $\approx 0.6158277481$ by Du [4]. Smith and MacGregor Smith have shown that the $\mathcal{R}$-sausage has produced the best known upper bound on the Steiner ratio in three dimensions [13].

Conjecture 1. The set of n-point $R$-sausages constitute the point configurations that in the limit, as $n$ approaches infinity, minimize the Steiner ratio for different dimensions.

Lemma 1. The infinite three-dimensional $R$-sausage contains within it a triple helix which passes through the fixed vertices that define the $R$-sausage. These points of the $R$-sausage are given by a recursive expression in the equation for some $\hat{a}, \hat{b}, \hat{c}, \hat{d} \in \Re$. These coefficients are $\left(\frac{2}{3}, \frac{2}{3}, \frac{2}{3},-1\right)$.

$$
V_{n}=\hat{a} V_{n-1}+\hat{b} V_{n-2}+\hat{c} V_{n-3}+\hat{d} V_{n-4}
$$

Proof. The $R$-sausage is generated by repeatedly reflecting a tetrahedral point in the facet opposite to it. The reflected point is chosen with respect to the Bucky-ball ${ }^{1}$ rule which is presented as Definition 2. The coordinates of the centroid $C$ of the triangle $V_{n-1}, V_{n-2}, V_{n-3}$ is given by $1 / 3\left(V_{n-1}+V_{n-2}+V_{n-3}\right)$. This expression can be derived by solving two pairs of simultaneous equations obtained from any two out of the three medians of the triangle intersecting at the point $C$. Let $r_{1}$ and $s_{1}$ be constants such that $r_{1}+s_{1}=1$ and $C=r_{1} V_{n-1}+s_{1} V_{n-2}$. Let $r_{2}$ and $s_{2}$ be constants such that $r_{2}+s_{2}=1$ and $C=r_{2} V_{n-2}+s_{2} V_{n-3}$. Equating these two expressions for $C$ one gets $r_{1} V_{n-1}+s_{1}\left(\frac{1}{2}\right)\left(V_{n-2}+V_{n-3}\right)=C=r_{2} V_{n-3}+s_{2}\left(\frac{1}{2}\right)\left(V_{n-1}+V_{n-2}\right)$ or $2 r_{1} V_{1}+s_{1} V_{2}+$ $s_{1} V_{3}=2 r_{2} V_{3}+s_{2} V_{1}+s_{2} V_{2}$. Comparing the coefficients of the vertices, we obtain $s_{1}=2 r_{2}, s_{2}=2 r_{1}$ and $s_{1}=s_{2}$ or $s_{1}=s_{2}=\frac{2}{3}$ and $r_{1}=r_{2}=\frac{1}{3}$. The position vector of $C$ is therefore $r_{1} V_{n-1}+s_{1}\left(\frac{1}{2}\right)\left(V_{n-2}+V_{n-3}\right)=1 / 3\left(V_{n-1}+2 / 3\left(\frac{1}{2}\right)\left(V_{n-2}+V_{n-3}\right)\right.$. That is $C=1 / 3\left(V_{n-1}+V_{n-2}+V_{n-3}\right)$. This is also the mid-point of the line joining the new vertex with the reflected point. Therefore $1 / 2\left(V_{n}+V_{n-4}\right)=C$ and $V_{n}=2 C-V_{n-4}$. That is, $V_{n}=2 / 3\left(V_{n-1}+V_{n-2}+V_{n-3}\right)-V_{n-4}$.

Identification of the optimal five, six, seven and larger point configurations which optimize the Steiner ratio in three dimensions for a point set of that size is formalized as Conjecture 2. A calculus of variations approach for analyzing a finite number of points in two dimensions was conducted by Rubinstein and Thomas [11]. The optimal four point configuration in three dimensions, with the optimal Steiner ratio, is the regular tetrahedron [13].

Conjecture 2. The set of n-point $R$-sausages constitute the configurations in three dimensions which achieve the optimal Steiner ratio, for a set of $n$ points in three dimensions.

\footnotetext{
${ }^{1}$ R. Buckminster Fuller developed the architectural construction known as the geodesic dome. This comprises a spidery network of interconnected tetrahedra (four-sided pyramids of equilateral triangles) forming a three-way, hemispherical grid that distributes stress evenly to all members of the entire structure and hence exhibits a high strength-to-weight ratio.
} 
Gilbert and Pollak also experimented with a network with movable Steiner vertices that are linked by unbending, untwisting rods to the fixed ends. Each rod will have a tensile force and they sought the network which would hold these tensile forces in equilibrium. Let $F_{1}, F_{2}, F_{3}, \ldots, F_{N}$ be unit forces acting at fixed vertices $V_{1}, V_{2}, V_{3}, \ldots, V_{N}$, respectively. They have proved the following theorem, which is critical to our argument [10]:

Theorem 3. If we draw unit vectors from a Steiner tree in the direction of each of the lines incident to $V_{1}, V_{2}, \ldots, V_{N}$ and let $F_{i}$ denote the sum of the unit vectors at $V_{i}$, then, in mechanical terms, $F_{i}$ is the external force needed at $V_{i}$ to hold the tree in equilibrium. The length of the tree $T$ has the simple dot product formula:

$$
T=V \cdot F \text {. }
$$

\section{Flat Sausage Development}

The flat sausage may be constructed by packing unit circles according to the Bucky-ball principle: start with a unit circle; add a circle next to the $\min (2, N-1)$ most recently added circle, and consider the centers of these circles as the point set.

Definition 3. The path topology for an $N$ point flat sausage is a full Steiner tree topology, with a total of $N-2$ Steiner points. Steiner point i is connected to $i+1$ for $i=1, \ldots, N-3$. Sausage point $\mathrm{i}$ is also attached to Steiner point $i-1$ for $i=2, \ldots, N-1$, and sausage point 1 is also attached to Steiner point 1 and sausage point $N$ is attached to Steiner point $N-2$.

Figure 1 shows six planar triangulations with the optimal topology. Counterclockwise from the top right-hand corner are featured tilings developing from a simple equilateral triangle to an eight point flat sausage with six Steiner points.

\section{Properties of the Flat Sausage}

One can draw a distinction between four rudimentary categories of Steiner topologies, on the basis of the type of the three adjacent vertices, that a Steiner vertex links to. This constitutes a finite alphabet, where each of the letters is a characterization of a Steiner point in terms of its neighboring vertices. In Fig. 2(a) we can see a type $S^{0}$ Steiner vertex. Figure 2(b)-(d) shows type $S^{1}, S^{2}$ and $S^{3}$ type Steiner vertices.

Lemma 2. The set of all Steiner vertices can be divided into four characteristic sets:

1. $S^{0}:$ Set of all Steiner vertices connected to three fixed vertices. This type of Steiner vertex arises in isolated triangles and at the beginning of the tree construction in other instances.

2. $S^{1}$ : Set of all Steiner vertices connected to two fixed vertices and one Steiner vertex.

3. $S^{2}$ : Set of all Steiner vertices connected to one fixed vertex and two Steiner vertices.

4. $S^{3}$ : Set of all Steiner vertices connected to three other Steiner vertices. 

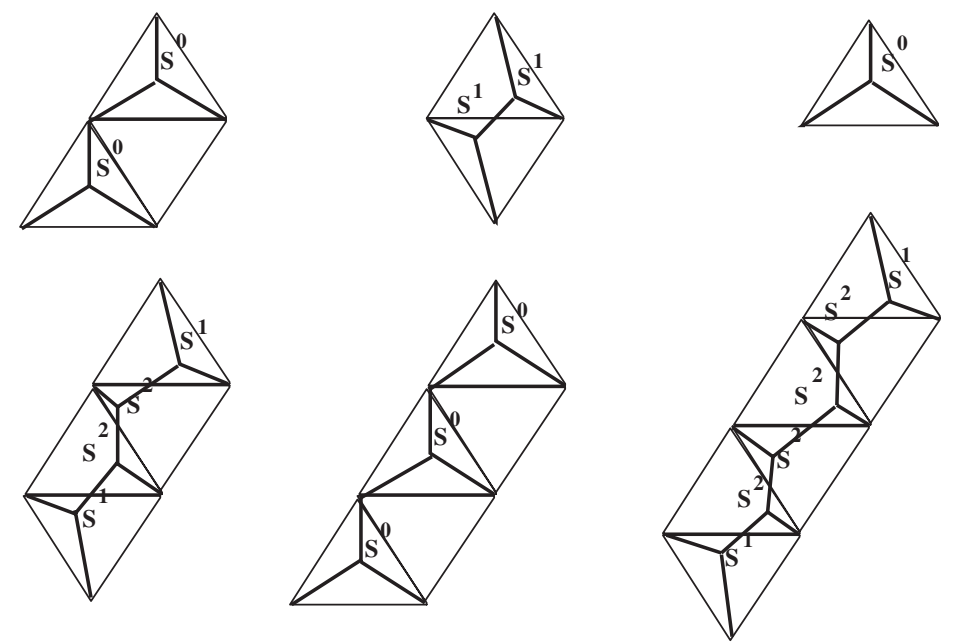

Fig. 1. Flat Sausage development.

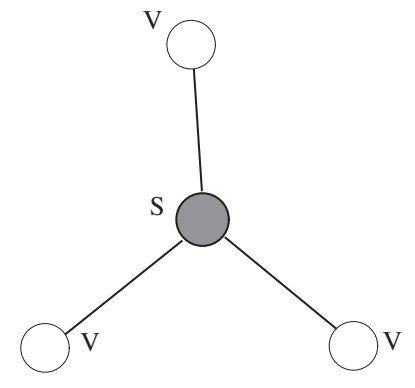

(a)

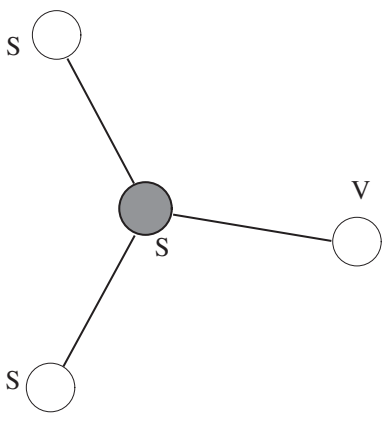

(c)

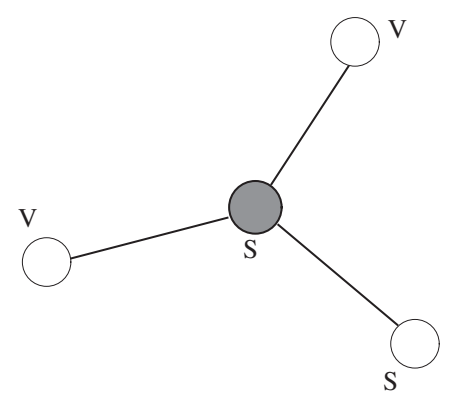

(b)

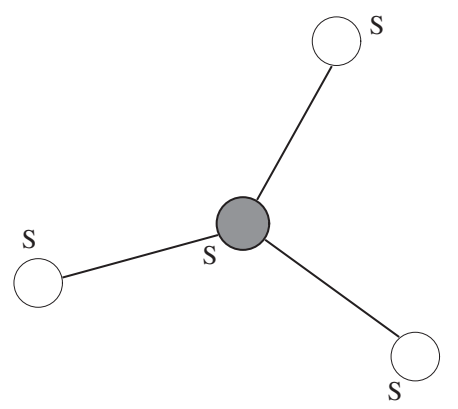

(d)

Fig. 2. The different types of Steiner vertices. 
Table 1. Steiner ratios for flat sausages with an even number of vertices.

\begin{tabular}{rrrcc}
\hline$N$ & MST length & SMT length & $\rho$ value & Time (s) \\
\hline 4 & 5.999868 & 5.291419 & 0.881923 & 0 \\
6 & 9.999780 & 8.717697 & 0.871789 & 0 \\
8 & 13.999692 & 12.165373 & 0.868974 & 1 \\
10 & 17.999604 & 15.620297 & 0.867813 & 0.0 \\
20 & 37.999164 & 32.923699 & 0.866432 & 0.0 \\
30 & 57.999590 & 50.239722 & 0.866208 & 0.0 \\
40 & 77.999150 & 67.557447 & 0.866131 & 0.0 \\
50 & 97.998710 & 84.876243 & 0.866096 & 0.0 \\
60 & 117.998270 & 102.195598 & 0.866077 & 0.0 \\
70 & 137.998696 & 119.516308 & 0.866068 & 0.0 \\
80 & 157.998256 & 136.836279 & 0.866062 & 0.0 \\
90 & 177.997816 & 154.156476 & 0.866058 & 0.0 \\
100 & 197.997376 & 171.476915 & 0.866057 & 0.1 \\
\hline
\end{tabular}

The path topology is equivalent to the Steiner set $\left\{\ldots, S^{2}, S^{2}, S^{2}, \ldots,\right\}$ in this convention.

Proof. This is straightforward. A Steiner point can have none, one, two or three Steiner point neighbors. This simple lemma is powerful because it enables one to make a complex taxonomical classification of any Steiner topology, as a permutation of these rudimentary topologies, for a specific traversal order.

Lemma 3. The Steiner ratio for the $n$ point flat sausage is $\rho_{2}=\sqrt{3} / 2$ as $n$ goes to infinity.

Proof. Table 1 presents the quantitative results from these experiments with the Branch and Bound algorithm, tailored specifically for the flat sausage. Figure 3 and Table 1 clearly reveal the convergence of $\rho_{2}=\sqrt{3} / 2 \approx 0.866$, as the number of vertices increases past 100 . The number theoretic argument for an odd number of vertices proceeds as follows: the size of the point set $n$ can be represented as $2 k+1$ for some positive integer $k$. Each of the triangles with indices $2 k-1,2 k, 2 k+1$ are equilateral and have an SMT consisting of three edges meeting at the centroid. Each of these triangles are conjoined at the $(2 k+1)$ st vertex. The $(2 k+1)$ st vertex for any value of $k$ is such that the internal angle subtended by the edges of the SMT is $2 \pi / 3$. The SMT for the flat sausage is equivalent to a $k$-fold version of the SMT for an equilateral triangle. The total SMT length is therefore $k \sqrt{3}$. The total MST length is $n-1$ or $2 k+1-1=2 k$. The $\rho$ is therefore the ratio of these two quantities, and equal to $\sqrt{3} / 2$. This is illustrated in Figs. 4 and 5 for nine and eleven vertices. $k$ ranges from $1, \ldots, 4$, and from $1, \ldots, 5$, respectively.

For flat sausages with an even number of vertices, we do not as yet have a simple number theoretic proof, and need to refer to work on $Z\left(P_{n}, \alpha\right)$ by Du et al. and independently by Smith [7], [12]. $Z\left(P_{n}, \alpha\right)$ is a zig-zag line where $P_{n}=\left\{p_{1}, p_{2}, \ldots, p_{n}\right\}$ denote the set of points, and $\alpha<180^{\circ}$ is the constant angle. The length of the SMT for 


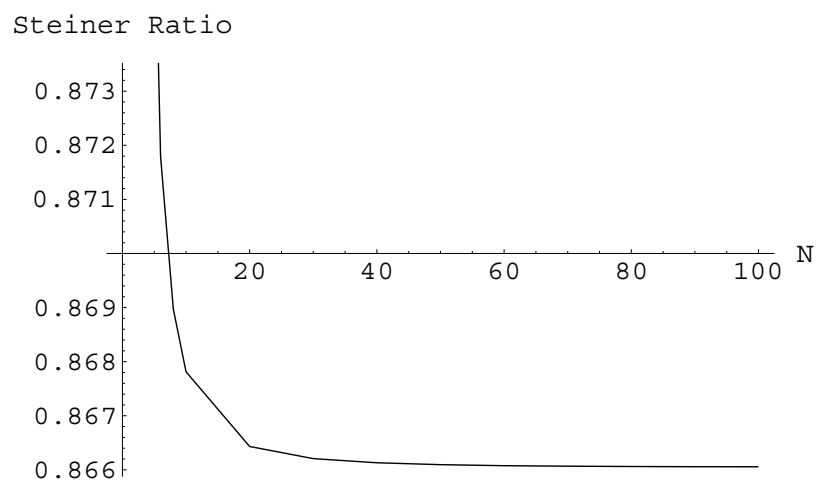

Fig. 3. Convergent Steiner ratio in two dimensions.

$\alpha=60^{\circ}$ is $\sqrt{3} k$ if $n$ is odd, and $\sqrt{\left[3\left(k^{2}+k\right)+1\right]}$ if $n$ is even. Again, on taking the limit as $k$ approaches infinity, we obtain a Steiner ratio of $\sqrt{3} / 2$.

Figures 6-10 on the other hand display four, six, eight and ten vertex flat sausages. Notice that the SMTs in these figures no longer have $n$-fold symmetry, and there are no degenerate Steiner points. They exhibit more clearly what we have defined as the path topology, although an analytic proof of this has been difficult. Lemma 4 shows how one may algebraically, construct the same flat sausage given an equilateral base triangle. Lemma 5 shows how one may derive the coordinates of all the Steiner vertices for a flat sausage, given the base triangle of Steiner vertices. These lemmas though consistent with a path topology are not a sufficient condition to conclude that the path topology is optimal for the flat sausage.

Lemma 4. The fixed vertices of the flat sausage are given by the recursive expression in (2) for some $\hat{a}, \hat{b}, \hat{c} \in \Re$. These coefficients are $(1,1,-1)$.

$$
V_{n}=\hat{a} V_{n-1}+\hat{b} V_{n-2}+\hat{c} V_{n-3} .
$$

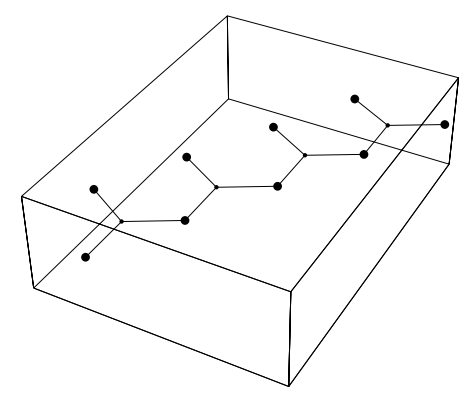

Fig. 4. SMT for the nine vertex flat sausage. 


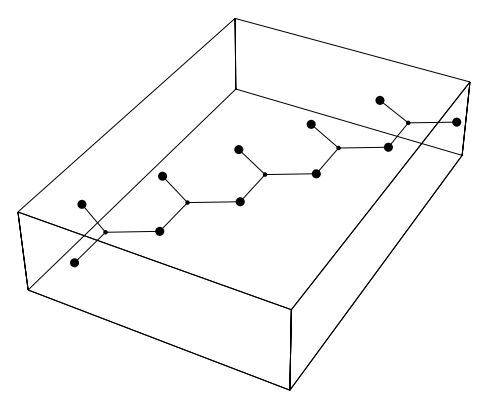

Fig. 5. SMT for the eleven vertex flat sausage.

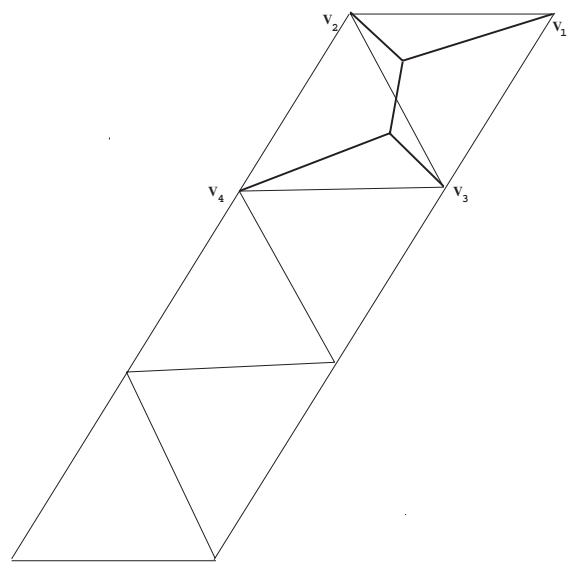

Fig. 6. Four vertex flat sausage.

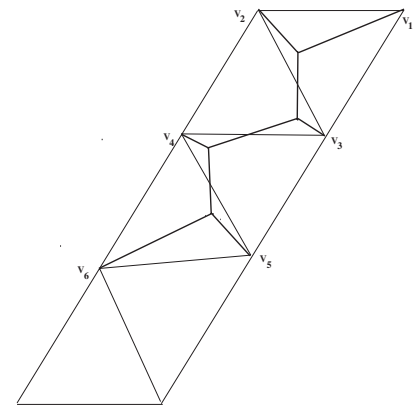

Fig. 7. Six vertex flat sausage. 


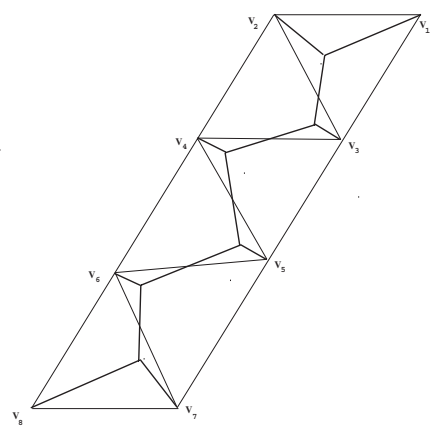

Fig. 8. Eight vertex flat sausage.

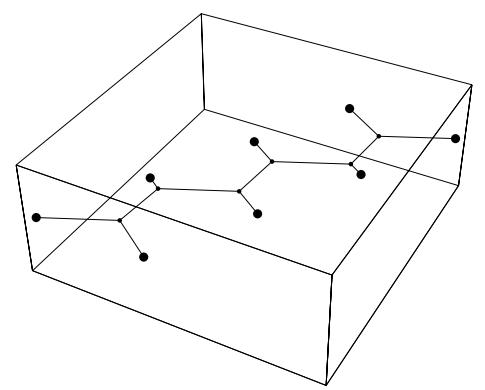

Fig. 9. Another view of the eight vertex flat sausage.

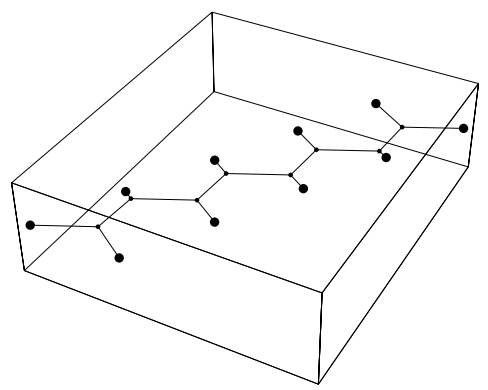

Fig. 10. A view of the ten vertex flat sausage.

Proof. From the parallelogram law of forces, $V_{n}+V_{n-3}=V_{n-1}+V_{n-2}$. Rewriting this as $V_{n}=V_{n-1}+V_{n-2}-V_{n-3}$ allows one to generate the successive vertices of the flat sausage. The vertices $V_{n-3}, V_{n-1}$ and $V_{n-2}$ have to be chosen so that the vertex $V_{n}$ is along the flat sausage. The first few vertices are generated as follows. $V_{0}$ is the reflected vertex and $V_{1}-V_{2}$ is the line of reflection. In the second iteration $V_{1}$ is the reflected vertex and $V_{3}-V_{2}$ the line of reflection. In the third iteration $V_{2}$ is the reflected vertex and $V_{3}-V_{4}$ is the line of reflection. In the $(N-1)$ st iteration $V_{n}$ is the reflected vertex 
and $V_{n+1}-V_{n+2}$ or $V_{n+2}-V_{n+1}$ is the line of reflection depending on whether $N$ is even or odd, respectively. Therefore $\hat{a}=\hat{b}=1$ and $\hat{c}=-1$.

Lemma 5. The Steiner vertices for the SMT of a two-dimensional flat sausage are given by the recursive expression in (3) for some $\bar{a}, \bar{b}, \bar{c} \in \Re$. These coefficients are $(1,1,-1)$.

$$
S_{n}=\bar{a} S_{n-1}+\bar{b} S_{n-2}+\bar{c} S_{n-3}
$$

Proof. The resultant of forces at each Steiner vertex is identical in an infinite flat sausage at equilibrium. The inter-site distances are therefore equal. That is,

$$
\overrightarrow{S_{i} S_{i+1}}=\left\|S_{i}-S_{i+1}\right\|=k_{1}, \quad \forall i
$$

and

$$
\overrightarrow{S_{i} S_{i+2}}=\left\|S_{i}-S_{i+2}\right\|=k_{2}, \quad \forall i
$$

Therefore as a sufficient condition of the parallelogram law of forces, $S_{i+3}+S_{i+2}=$ $S_{i}+S_{i+1}$ or $S_{i+3}=S_{i}+S_{i+1}-S_{i+2}$.

\subsection{Flat Sausages Have the Path Topology}

Though we have developed a formula for enumerating the successive Steiner points for the flat sausage, we have not yet proved that the flat sausage has the path topology. The proof proceeds in the following way: The minimizing direction of the objective function, the length of the SMT, is shown to be the negative gradient of the objective function, which is towards the centroid of the triangle. Next two flat sausage elements are combined by conjoining their outermost edges. The triangle formed by this common vertex and the two associated Steiner points is much like the case of the equilateral triangle. The corner of the triangle common to both edges is a point of non-differentiability. The left- and righthand gradient $\nabla L(X, Y)_{\delta h \rightarrow 0+}=\left[\partial L / \partial X_{\delta h \rightarrow 0+}, \partial L / \partial Y_{\delta h \rightarrow 0+}\right]$ and $\nabla L(X, Y)_{\delta h \rightarrow 0-}=$ $\left[\partial L / \partial X_{\delta h \rightarrow 0-}, \partial L / \partial Y_{\delta h \rightarrow 0-}\right]$ do exist at this point [11]. After a split operation at this vertex, the locus of the resulting Steiner point is in the opposite direction of the derivative, towards the axis of the composite flat sausage instead of the centroid of a lone triangle. There are two minimizing directions, and both vectors can be resolved parallel and perpendicular to the edge across this vertex. The resultant of the forces parallel to the edge cancel each other out. The forces perpendicular to the edge reinforce each other and are towards the centroid of the triangle.

This split operation, and consequent optimization, does not disrupt the path topology in the neighborhood of the triangle, as the negative gradient vector(s) do not cross any other edge during this operation. Therefore this process can be repeated ad infinitum to generate the entire semi-group and the path topology for the infinite flat sausage.

Lemma 6. If the orientation of the elements (terminals) follows the path topology, then the binary composition, consisting of a vertex split, and the subsequent relaxed 
iteration as applied to flat sausages is well defined and causes the inclusion of exactly one additional Steiner vertex into the path topology.

Proof. Let $V_{1}^{i}, V_{2}^{i}, \ldots, V_{n_{1}}^{i}$ denote the vertices of the flat sausage element $a_{i}$. Let $S_{1}^{i}$, $S_{2}^{i}, \ldots, S_{n_{1}-2}^{i}$ denote the Steiner vertices of this element. Let $V_{1}^{j}, V_{2}^{j}, \ldots, V_{n_{2}}^{j}$ denote the vertices of the $\mathcal{R}$-sausage $a_{j}$. Let $S_{1}^{j}, S_{2}^{j}, \ldots, S_{n_{2}-2}^{j}$ denote the Steiner vertices of this element. The angle subtended by the last Steiner vertex $S_{n_{1}-2}^{i}$ in the element $a_{i}$ and the leading Steiner vertex $S_{1}^{j}$ of the element $a_{j}$ at the vertex $V_{n_{1}}$ is less than $2 \pi / 3$. The negative derivative at this vertex is normal to the orientation of the composite element. This centripetal force at the Steiner vertex, when transmitted to the Steiner vertices of the two elements, remains perpendicular to the orientation of the elements. For this reason, the drift towards the equilibrium position of the sum, which is guided by the relaxed iteration will not lead to an alternate topology. The binary composition is therefore well defined. Figure 11 illustrates this argument for three sausage elements, considered individually at the top of the figure. The first element is the six point sausage, and the second is a five point sausage. Two points with an edge between them are also included in this set to simulate the situation of adding one additional point to an existing sausage
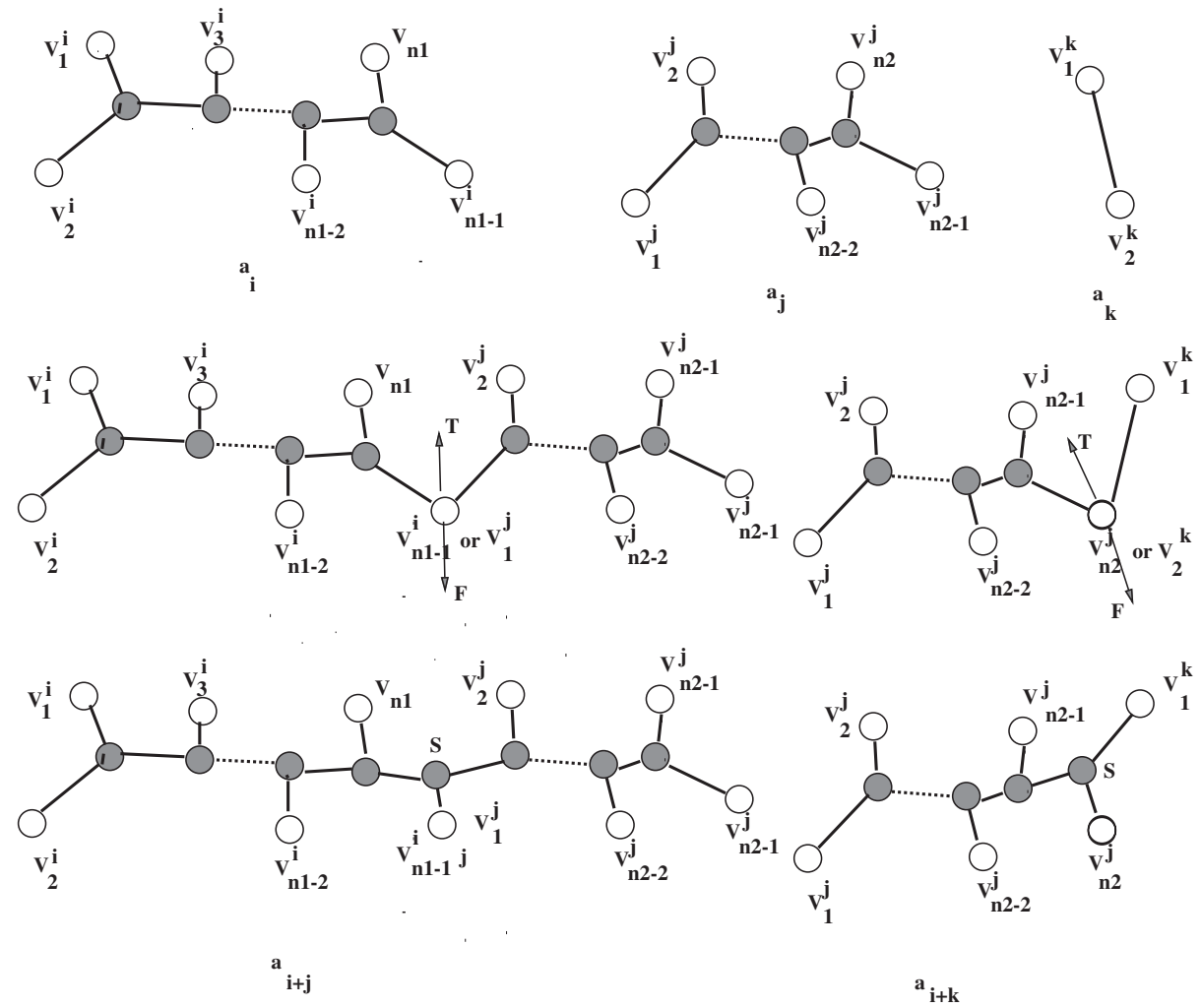

Fig. 11. The binary composition for flat sausages. 
set. According to Du and Smith, the $d$-sausages may not support such operations in dimensions higher than three [6].

Definition 4. A set of elements $G$, together with a binary composition + , is called an Abelian group, denoted $\langle G,+\rangle$, if it satisfies the following group axioms:

1. $\forall a, b \in G, a+b \in G$.

2. $\forall a, b \in G, a+b=b+a$.

3. $\forall a, b, c \in G,(a+b)+c=a+(b+c)$.

4. There exists an element $0 \in G$ such that $a+0=0+a=a, \forall a \in G$.

5. For every $a \in G$ there exists a $b \in G$ such that $a+b=0$. This element is called the inverse of element a and is denoted $-a$.

Remark 4. If all the above axioms are satisfied for a particular set and composition, except for axiom 5, the pair $\langle G,+\rangle$ is called an Abelian semi-group. If axiom 2 also does not hold, then the pair $\langle G,+\rangle$ is simply called a semi-group.

Lemma 7. The set of all flat sausages of finite length, in three dimensions, constitute an infinite abelian semi-group that is closed under a binary composition. The topology exhibited by a typical element of this semi-group is called the path topology.

Proof. We have shown that the binary composition defined in Lemma 6 is well defined on the set of all flat sausages. Under this composition, they satisfy axioms 1-4 of Definition 4. It is however not a group as there is no inverse for a flat sausage. This semi-group may also be denoted $\left\langle a_{n}\right\rangle$ or $\left\langle a_{1}^{n}\right\rangle$ as every element except the identity element can be generated from $a_{1}$ by applying the binary composition, one or more times, to a unit element:

$$
\begin{gathered}
a_{1}=a_{1}=a_{1}^{1}, \\
a_{2}=a_{1}+a_{1}=a_{1}^{2}, \\
a_{3}=a_{1}^{2}+a_{1}=a_{1}+a_{1}+a_{1}=a_{1}^{3}, \\
a_{4}=a_{1}^{3}+a_{1}=a_{1}+a_{1}+a_{1}+a_{1}=a_{1}^{4}, \\
a_{n}=a_{1}^{n-1}+a_{1}=\underbrace{a_{1}+a_{1}+a_{1} \cdots+a_{1}}_{n-1 \text { terms }}+a_{1}=a_{1}^{n} .
\end{gathered}
$$

Note that by $a_{1}^{n}$ one means a Maxwell sum of $n$ terms rather than a product. Observe the nice factorization of the Maxwell sums. Figure 12 illustrates the first few members of this infinite semi-group.

Theorem 5. The infinite flat sausage in $E^{2}$ has the path topology.

Proof. By applying the binary composition defined in Lemma 6 a countably infinite number of times, the infinite flat sausage is also a member of the semi-group defined in Lemma 7. 


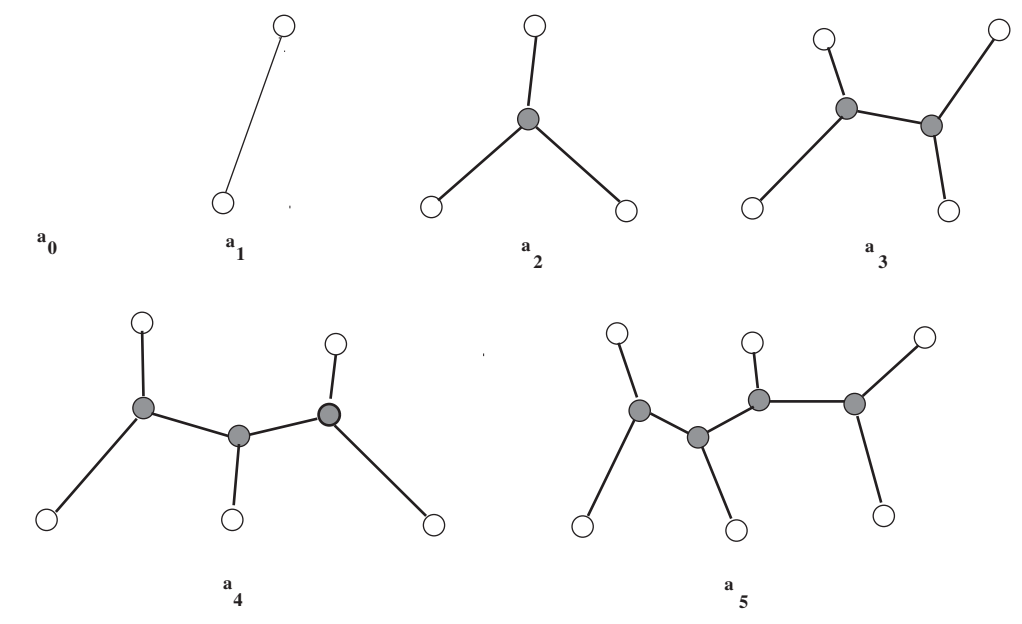

Fig. 12. Members of the infinite semi-group.

\section{Properties of the $\mathcal{R}$-Sausage}

There are many other interesting conjectures about the three-dimensional $\mathcal{R}$-sausage. These are primarily concerned with the structure of the $\mathcal{R}$-sausage. In this section we address some of these issues.

Figure 13 reveals the monotonic decrease in the Steiner ratio in three dimensions. A modular study of the structure and evolving geometry of the $\mathcal{R}$-sausage was conducted to gather more evidence. Figure 14 illustrates the three-dimensional structure of the $\mathcal{R}$-sausage along with its convex hull [13].

The $\mathcal{R}$-sausage was computationally rotated and translated by applying a transformation matrix, so that its axis, derived from the centroids of the constituent tetrahedra, was approximately perpendicular to the plane. Given this best transformation matrix, for an $\mathcal{R}$-sausage of over 1000 tetrahedra, the 31 st vertex was found offset from the first

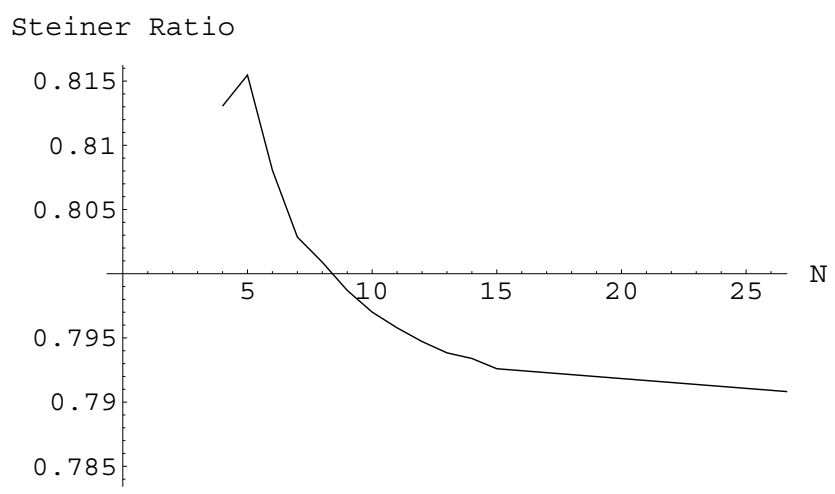

Fig. 13. Convergent Steiner ratio in three dimensions. 


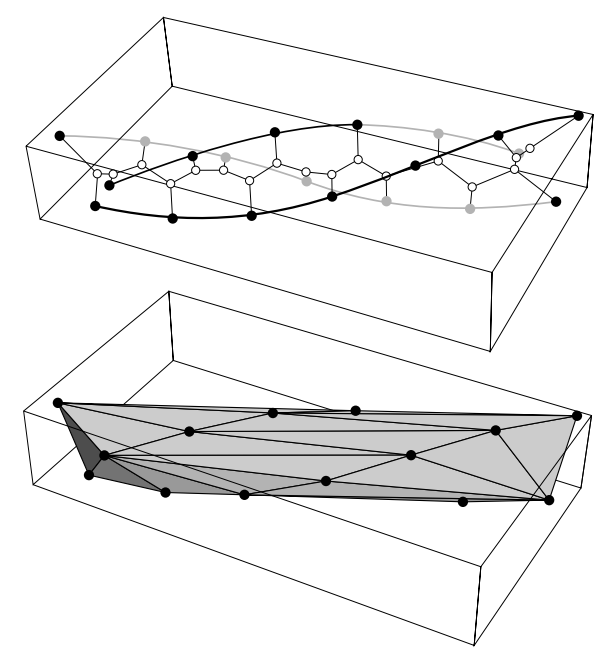

Fig. 14. The $\mathcal{R}$-sausage and its convex hull.

vertex by approximately $\frac{5}{360} \pi$. This confirmed Coxeter's work with 28 tetrahedra. The $\mathcal{R}$-sausage does not have a fixed periodicity, even though it has a recursive structure. This axial view phenomenon in its early stages is visible in Figure 15, which has been obtained as the SMT for a very long, though finite, $\mathcal{R}$-sausage vertex set.

Further computational assessment of the internal structure of a finite length threedimensional $\mathcal{R}$-sausage with unit edge length was conducted. The fixed and Steiner vertices were grouped into the following chains:

Chain A: $\left(V_{1}, V_{4}, V_{7}, V_{10}, V_{13}, V_{16}, V_{19}, V_{22}, \ldots\right)$,

Chain B: $\left(V_{2}, V_{5}, V_{8}, V_{11}, V_{14}, V_{17}, V_{20}, V_{23}, \ldots\right)$,

Chain C: $\left(V_{3}, V_{6}, V_{9}, V_{12}, V_{15}, V_{18}, V_{21}, V_{24}, \ldots\right)$,

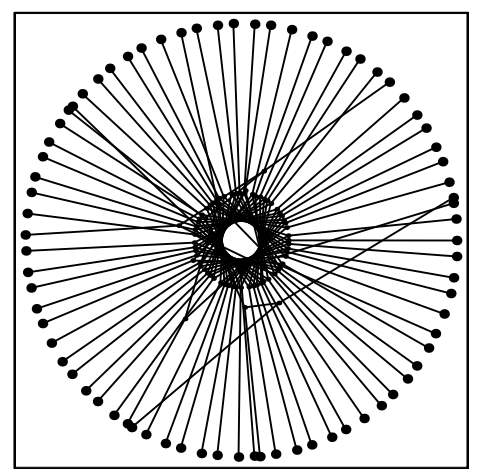

Fig. 15. End view of $\mathcal{R}$-sausage. 


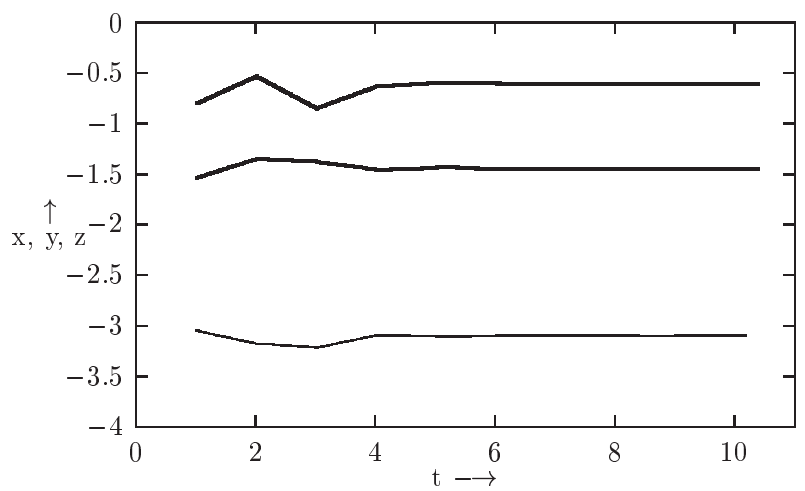

$\mathrm{x}$ coord

y coord.

Fig. 16. Coordinates of Steiner vertex $S_{0}$.

Chain a: $\left(S_{0}, S_{3}, S_{6}, S_{9}, S_{12}, S_{15}, S_{18}, S_{21}, \ldots\right)$,

Chain b: $\left(S_{1}, S_{4}, S_{7}, S_{10}, S_{13}, S_{16}, S_{19}, S_{22}, \ldots\right)$,

Chain c: $\left(S_{2}, S_{5}, S_{8}, S_{11}, S_{14}, S_{17}, S_{20}, S_{23}, \ldots\right)$.

Consider the first four Steiner vertices, $S_{0}-S_{3}$, corresponding to the optimal solution of a finite length $\mathcal{R}$-sausage. Plots such as those displayed in Figs. 16-19 showed that the coordinates of the first few Steiner vertices begin to stabilize around particular values, as the number of vertices in the $\mathcal{R}$-sausage increases. Steiner vertices $S_{0}$ and $S_{3}$ are on chain a, Steiner vertex $S_{1}$ is on chain b, and Steiner vertex $S_{2}$ is on chain c. This constituted the initial evidence that the locus of the Steiner vertices converges over time and yields recursive or iterative coefficients.

One may wish to express $S_{4}$ as a convex combination of $S_{0}, S_{1}, S_{2}, S_{3}$. Numerical analysis methods suggested solving a linear system of equations for the interpolating variables $X_{1}, X_{2}, X_{3}$ and $X_{4}$. The coefficients of the following equations are the Euclidean

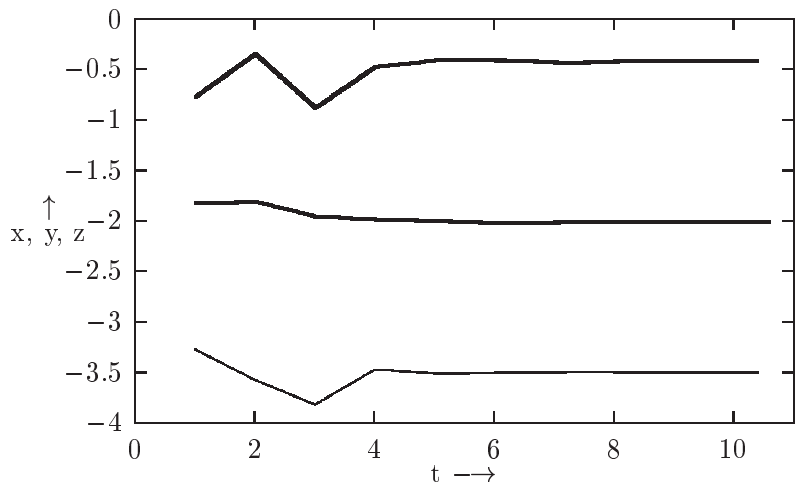

$\mathrm{x}$ coord -

z-coord

Fig. 17. Coordinates of Steiner vertex $S_{1}$. 


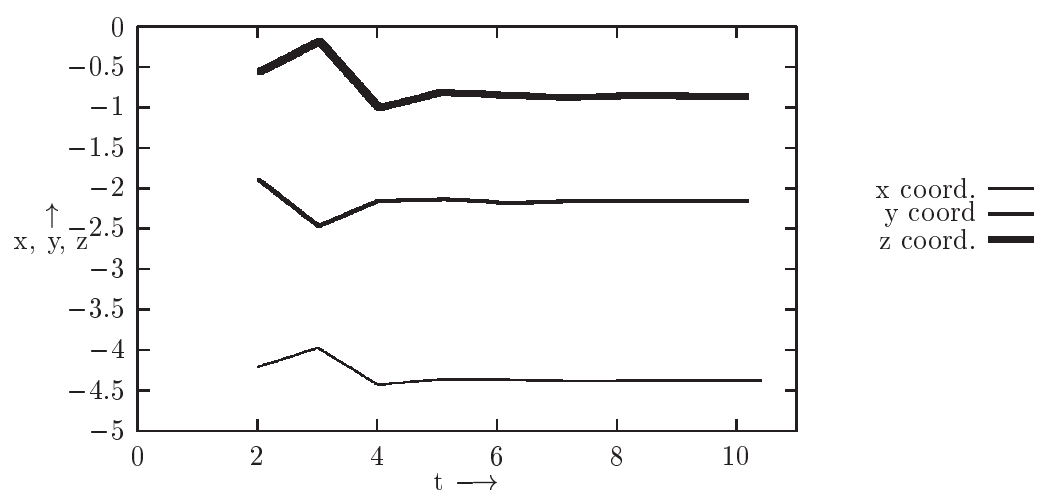

Fig. 18. Coordinates of Steiner vertex $S_{2}$.

coordinates of the first four Steiner vertices which had been calculated by the Branch and Bound algorithm:

$$
\begin{gathered}
-3.095960 X_{1}-3.498365 X_{2}-4.374615 X_{3}-5.320049 X_{4}=-5.860865 \\
-1.448044 X_{1}-2.015002 X_{2}-2.160861 X_{3}-2.421030 X_{4}=-3.274276 \\
-0.606943 X_{1}-0.424854 X_{2}-.861840 X_{3}-0.211866 X_{4}=-0.408658 \\
1.000000 X_{1}+1.000000 X_{2}+1.000000 X_{3}+1.000000 X_{4}=1.0000000
\end{gathered}
$$

That is,

$$
X_{1} S_{0}+X_{2} S_{1}+X_{3} S_{2}+X_{4} S_{3}=S_{4},
$$

where

$$
X_{1}+X_{2}+X_{3}+X_{4}=1 \text {. }
$$

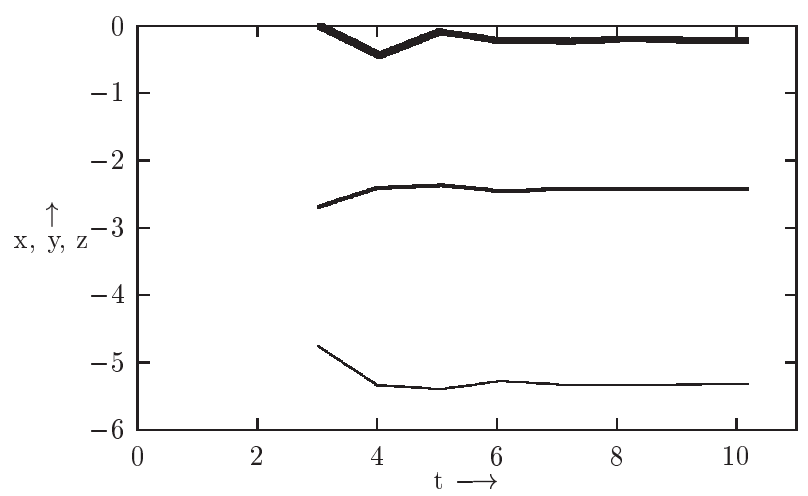

$$
\begin{aligned}
& \mathrm{x} \text { coord. }- \\
& \mathrm{y} \text { coord }- \\
& \mathrm{z} \text { coord. }
\end{aligned}
$$

Fig. 19. Coordinates of Steiner vertex $S_{3}$. 
Table 2. Cylindrical coordinates of the fixed vertices in three-dimensional $\mathcal{R}$-sausages.

\begin{tabular}{cccrc}
\hline Fixed vertices & Twist angle (radians) & Twist angle (degrees) & \multicolumn{1}{c}{ Radius } & \multicolumn{1}{c}{$Z$-axis } \\
\hline 100 & 0.464538 & 26.616087 & 46.533745 & -0.414621 \\
200 & 0.464139 & 26.593238 & 91.245387 & -0.403004 \\
300 & 0.463952 & 26.582509 & 135.962945 & -0.401460 \\
400 & 0.463884 & 26.578648 & 180.682234 & -0.403862 \\
500 & 0.463836 & 26.575897 & 225.402276 & -0.400075 \\
600 & 0.463802 & 26.573911 & 270.122714 & -0.402008 \\
700 & 0.463783 & 26.572854 & 314.843390 & -0.401359 \\
800 & 0.463764 & 26.571739 & 359.564223 & -0.400208 \\
900 & 0.463752 & 26.571037 & 404.285162 & -0.401782 \\
1000 & 0.463742 & 26.570477 & 449.006179 & -0.400220 \\
\hline
\end{tabular}

This linear system can be represented compactly in matrix notation as $P X=b$ where $P$ is a $4 \times 4$ matrix and $X$ and $b$ are $4 \times 1$ column vectors. The coefficients on the left-hand side of the equations are the entries of matrix $P$, and the coefficients on the right-hand side of the equations are the entries of the column vector $b$. The inverse of $P$ is $P^{-1}$ and $P^{-1} b=X$. The above linear system can be solved by reducing an identity matrix if the determinant is non-singular. On doing so, one obtains the values of the coefficients. Similar experiments were conducted with the other sets of Steiner vertices along the $\mathcal{R}$-sausage SMT. The coefficients converge to $(-0.99,0.67,0.67,0.67)$. The inverse computations were performed correct to six decimal places and rounded off to the second decimal place. Tables 2-5 display the cylindrical coordinates and the modular difference among successive vertices of the $\mathcal{R}$-sausage and its corresponding SMT. These computed results re-affirmed the hypothesis that the locus of the Steiner vertices was also a helix, and that the recursive coefficients $\hat{a}, \hat{b}, \hat{c}$ and $\hat{d}$ are $\left(-1, \frac{2}{3}, \frac{2}{3}, \frac{2}{3}\right)$. Lemma 8 consolidates all this evidence, with a vector algebraic proof.

Lemma 8. The coordinates of the Steiner vertices in the three-dimensional $\mathcal{R}$-sausage are given by the recursive expression in (4) for some $\bar{a}, \bar{b}, \bar{c}, \bar{d} \in \Re$. These coefficients

Table 3. Gap between the cylindrical coordinates of the fixed vertices in three-dimensional $\mathcal{R}$-sausages.

\begin{tabular}{ccccc}
\hline Fixed vertices & Twist angle (radians) & Twist angle (degrees) & Radius & $Z$-axis \\
\hline 100 & -0.003355 & -0.192278 & 0.884360 & 0.005041 \\
200 & -0.001568 & -0.089872 & 0.889379 & 0.005085 \\
300 & -0.001083 & -0.062107 & 0.891047 & 0.008019 \\
400 & -0.000804 & -0.046106 & 0.891888 & -0.00505 \\
500 & -0.000639 & -0.036614 & 0.892398 & 0.004687 \\
600 & -0.000540 & -0.030984 & 0.892738 & 0.001851 \\
700 & -0.000456 & -0.023079 & 0.892978 & 0.000607 \\
800 & -0.000402 & -0.023079 & 0.893158 & 0.003365 \\
900 & -0.000358 & -0.020535 & 0.893299 & -0.000047 \\
1000 & -0.000320 & -0.018347 & 0.893412 & 0.001816 \\
\hline
\end{tabular}


Table 4. Cylindrical coordinates of the Steiner vertices in three-dimensional $\mathcal{R}$-sausages.

\begin{tabular}{cccrc}
\hline Steiner vertices & Twist angle (radians) & Twist angle (degrees) & \multicolumn{1}{c}{ Radius } & \multicolumn{1}{c}{$Z$-axis } \\
\hline 98 & 0.463761 & 26.571599 & 46.511136 & -0.411359 \\
198 & 0.463747 & 26.570761 & 91.232133 & -0.402239 \\
298 & 0.463700 & 26.568091 & 135.953344 & -0.401027 \\
398 & 0.463693 & 26.567689 & 180.674621 & -0.402814 \\
498 & 0.463685 & 26.567243 & 225.395927 & -0.400061 \\
598 & 0.463677 & 26.566742 & 270.117249 & -0.401409 \\
698 & 0.463675 & 26.566651 & 314.838581 & -0.400994 \\
798 & 0.463670 & 26.566352 & 359.559919 & -0.401249 \\
898 & 0.463669 & 26.566303 & 404.281261 & -0.401249 \\
998 & 0.463667 & 26.566208 & 449.002607 & -0.400160
\end{tabular}

are precisely $\left(-1, \frac{2}{3}, \frac{2}{3}, \frac{2}{3}\right)$.

$$
S_{n}=\bar{a} S_{n-1}+\bar{b} S_{n-2}+\bar{c} S_{n-3}+\bar{d} S_{n-4} .
$$

Proof. To some extent this is similar to the case of the flat sausage. The inter-site distances between the Steiner vertices are equal, because the resultant of all the forces at a Steiner vertex in the infinite $\mathcal{R}$-sausage at equilibrium is the same. That is,

$$
\overrightarrow{S_{i} S_{i+1}}=\left\|S_{i}-S_{i+1}\right\|=k_{1}, \quad \forall i,
$$

and

$$
\overrightarrow{S_{i} S_{i+2}}=\left\|S_{i}-S_{i+2}\right\|=k_{2}, \quad \forall i
$$

Furthermore,

$$
\overrightarrow{S_{i} S_{i+3}}=\left\|S_{i}-S_{i+3}\right\|=k_{3}, \quad \forall i .
$$

Even though the base tetrahedron is no longer regular, the position vector argument, that was employed in the proof of Lemma 5, continues to hold:

$$
\frac{1}{2}\left(S_{i-1}+S_{i}\right)=\frac{1}{3}\left(S_{i-4}+S_{i-3}+S_{i-2}\right), \quad \forall i .
$$

Table 5. Gap between the cylindrical coordinates of Steiner vertices in three-dimensional $\mathcal{R}$ sausages.

\begin{tabular}{ccccr}
\hline Steiner vertices & Twist angle (radians) & Twist angle (degrees) & Radius & \multicolumn{1}{c}{$Z$-axis } \\
\hline 98 & -0.000270 & -0.015475 & 0.879492 & -0.000188 \\
198 & 0.001568 & 0.089872 & 0.889379 & 0.005085 \\
298 & 0.000091 & 0.005269 & 0.889564 & 0.000621 \\
398 & 0.000072 & 0.004139 & 0.890786 & 0.000178 \\
498 & 0.000057 & 0.003287 & 0.891526 & 0.000851 \\
598 & 0.000047 & 0.002730 & 0.892011 & 0.000006 \\
698 & 0.000041 & 0.002382 & 0.892357 & 0.000409 \\
798 & 0.000035 & 0.002038 & 0.892616 & 0.000351 \\
898 & 0.000032 & 0.001872 & 0.892824 & 0.000007 \\
988 & 0.000029 & 0.001680 & 0.892984 & 0.000425 \\
\hline
\end{tabular}


Rearranging terms:

$$
S_{i}=\frac{2}{3}\left(S_{i-4}+S_{i-3}+S_{i-2}\right)-S_{i-1}, \quad \forall i
$$

The reflective symmetry that is exhibited there is inherited, in the limit, by this triangulation of the optimal Steiner vertices.

Conjecture 6. Let the $V_{i}$ 's denote the coordinates of the fixed vertices. The $S_{i}$ 's are the coordinates of the movable Steiner vertices. The $l_{i}$ 's and the $l_{i}^{\prime}$ 's are the absolute values of the inter-site distances between the fixed vertices and the Steiner vertices. The $d_{i}$ 's and the $d_{i}^{\prime}$ 's are the inter-site distances between the Steiner vertices. One can now formulate the $\mathcal{R}$-sausage as a non-linear programming problem:

$$
\begin{aligned}
& V_{i}=\left(a_{i}, b_{i}, c_{i}\right), \quad \forall i=2, \ldots, n, \\
& S_{i}=\left(x_{i}, y_{i}, z_{i}\right), \quad \forall i=2, \ldots, m, \\
& l_{i}=\left\|V_{i}-S_{i-1}\right\|, \quad \forall i=2, \ldots,\lceil m / 2\rceil+1, \\
& l_{i}^{\prime}=\left\|V_{n+1-i}-V_{n-i}\right\|, \quad \forall i=2, \ldots,\lceil m / 2\rceil+1 \text {, } \\
& d_{i}=\left\|S_{i}-S_{i+1}\right\|, \quad \forall i=1, \ldots,\lceil m / 2\rceil-1, \\
& d_{i}^{\prime}=\left\|S_{m+1-i}-S_{m-i}\right\|, \quad \forall i=1, \ldots,\lceil m / 2\rceil-1 \text {, } \\
& l_{1}=\left\|V_{1}-S_{1}\right\| \quad \text { and } \quad\left\|l_{1}^{\prime}=\right\| V_{n}-S_{m} \| \text {. }
\end{aligned}
$$

Then

$$
\text { Minimize } \sum_{i}^{m / 2+1} l_{i}+\sum_{i}^{m / 2-1} d_{i}
$$

such that

$$
\begin{aligned}
l_{i}=l_{i}^{\prime}, & \forall i=1, \ldots,\lceil m / 2\rceil+1, \\
d_{i}=d_{i}^{\prime}, & \forall i=1, \ldots,\lceil m / 2\rceil-1 .
\end{aligned}
$$

These topologies are portrayed in Fig. 20. Table 6 presents the mean inter-site distances, and mean vertex to axis distance in Cartesian coordinates, with computed evidence in support of this conjecture.

In general, if $n$ is even, then there are $n / 2-1$ different SMT topologies. For an odd number of vertices there are $(n-1) / 2-1$ different topologies. For each topology, one can also enumerate the Steiner base set. For the six vertex set in Fig. 21, one topology has the Steiner base set $\left\{S^{1}, S^{2}, S^{2}, S^{1}\right\}$ and the other topology has the Steiner base set $\left\{S^{3}, S^{1}, S^{1}, S^{1}\right\}$. Though one has enumerated the SMT topologies, and the convex hulls for a finite, regular point set, it is a complex task to find out which SMT is optimal for a particular convex hull. Proving that the path topology corresponding to the set $\left\{\ldots, S^{2}, S^{2}, S^{2}, \ldots\right\}$ is optimal for the $\mathcal{R}$-sausage structure has proved a signifantly complicated exercise. It is not yet clear how one can, if at all, put the remaining SMT topologies into a one-one correspondence with the other convex hulls. An algebraic, though non-linear, expression in terms of vector products has been obtained by resolving all the forces exerted on a particular Steiner vertex in the optimal topology for the infinite 

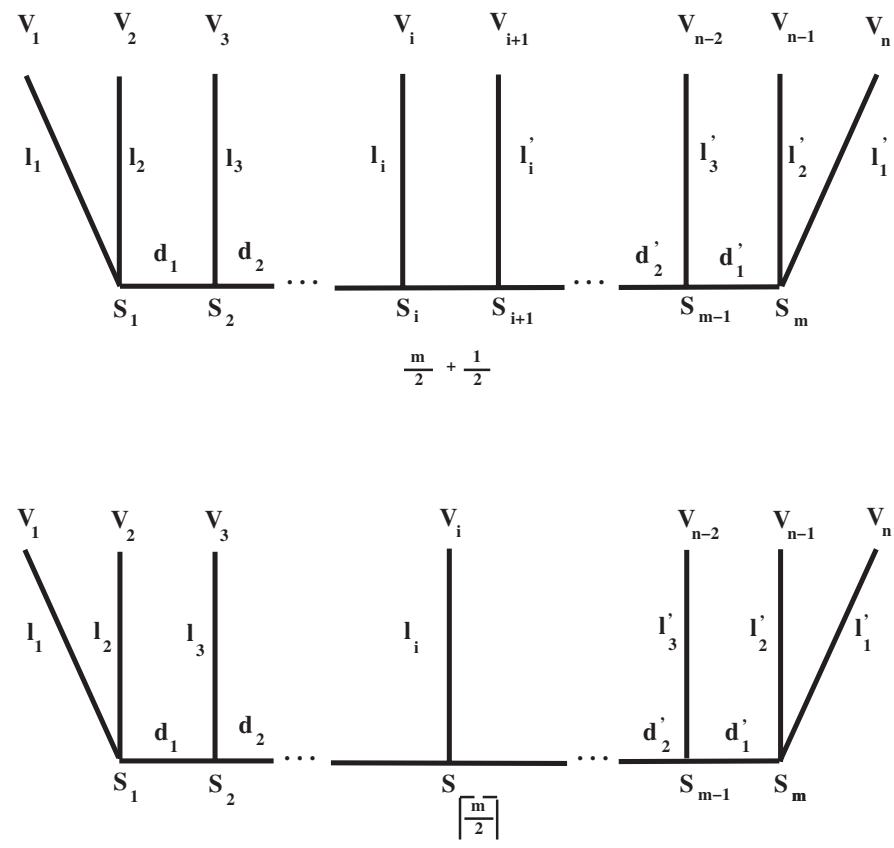

Fig. 20. Even- and odd-numbered topologies.

$\mathcal{R}$-sausage, and summing over all the Steiner vertices. At equilibrium, by Maxwell's theorem, the following vector sums should be zero:

$$
\begin{array}{r}
\sum_{i=1}^{\infty}\left(2 . \overrightarrow{S_{i} S_{i+1}} \cos \pi / 3+\overrightarrow{S_{i} V_{i-1}}\right)=0, \\
\sum_{i=1}^{\infty}\left(\overrightarrow{S_{i} S_{i+1}}+\overrightarrow{S_{i} V_{i-1}}\right)=0 .
\end{array}
$$

We have found compact algebraic representations for them as infinite sums. The solution of this objective function is feasible only for small vertex sets due to the extreme non-linearity and non-differentiability of the objective function.

Table 6. Inter-site and radial distances within a threedimensional $\mathcal{R}$-sausage.

\begin{tabular}{ccc}
\hline Type of vertex & Type of vertex & Inter-site distance \\
\hline Fixed & Fixed & 1.000 \\
Steiner & Steiner & 1.0690 \\
Steiner & Fixed & 1.1490 \\
Steiner & Axis & 0.320 \\
Fixed & Axis & 1.469 \\
\hline
\end{tabular}



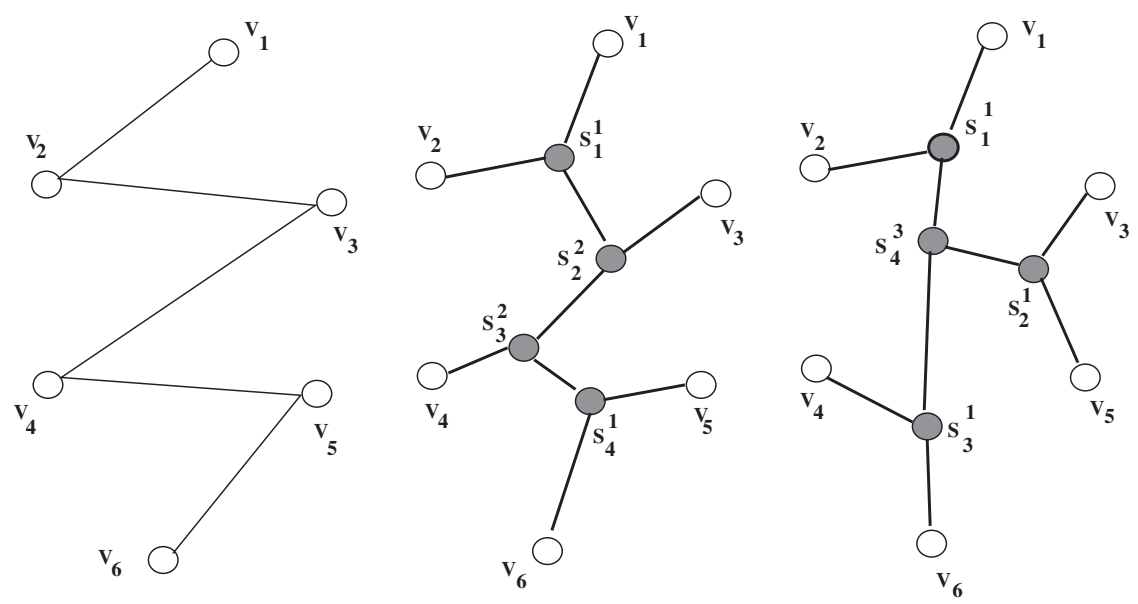

Fig. 21. MST and SMTs for six vertices.

\subsection{R-Sausages Have the Path Topology}

It is our concern to show that, given the regular tetrahedra provision on the vertex sets above, the infinite $\mathcal{R}$-sausage in $E^{3}$ also has the path topology. Different length threedimensional $\mathcal{R}$-sausages have been shown to possess the path topology by complete enumeration. Computational experiments show that $\rho_{3} \approx 0.784$, the conjectured optimal $\rho_{3}$. This convergence of the $\rho$ values of these finite length $\mathcal{R}$-sausages is no guarantee that the path topology is the optimal topology for the infinite $\mathcal{R}$-sausage. Tables 7 and 8 provide computational evidence, from early attempts, to ascertain if a topology other than the path topology could have a lower or equal $\rho$ value for the $\mathcal{R}$-sausage. The first two columns of these two tables hold the values of $i$ and $j$. The other columns provide the SMT length and the $\rho_{2}$ value. For a six vertex set, the path topology is the one pictured in the middle, and the alternative topology is the one on the extreme right, in Fig. 21. The moving indices $i$ and $j$ correspond to fixed vertices $V_{1}$ and $V_{2}$ in these two topologies. For $(i, j)=(1,2)$, one obtains the path topology, with the lowest $\rho_{3}$. Thus the path topology is superior to the non-path topology for a six vertex three-dimensional vertex set. As

Table 7. SMT lengths and $\rho$ values for path topology.

\begin{tabular}{cccccccc}
\hline$i$ & $j$ & SMT length & $\rho$ & $i$ & $j$ & SMT length & $\rho$ \\
\hline 1 & 2 & 11.4278 & 0.808065 & 2 & 6 & 12.3196 & 0.871127 \\
1 & 3 & 11.5292 & 0.815235 & 3 & 4 & 11.8647 & 0.838960 \\
1 & 4 & 11.5292 & 0.815235 & 3 & 5 & 12.0101 & 0.849245 \\
1 & 5 & 12.0565 & 0.852520 & 3 & 6 & 11.9588 & 0.845611 \\
1 & 6 & 12.3196 & 0.871127 & 4 & 5 & 12.0101 & 0.849245 \\
2 & 3 & 11.5641 & 0.871127 & 4 & 6 & 11.9588 & 0.845611 \\
2 & 4 & 11.5641 & 0.817704 & 5 & 6 & 11.8872 & 0.840555 \\
2 & 5 & 11.8891 & 0.840690 & $*$ & $*$ & $*$ & $*$ \\
\hline
\end{tabular}


Table 8. SMT lengths and $\rho$ values for non-path topology.

\begin{tabular}{cccccccc}
\hline$i$ & $j$ & SMT length & $\rho$ & $i$ & $j$ & SMT length & $\rho$ \\
\hline 1 & 2 & 11.6040 & 0.820527 & 2 & 6 & 12.0950 & 0.855246 \\
1 & 3 & 11.7371 & 0.829940 & 3 & 4 & 11.6040 & 0.820527 \\
1 & 4 & 11.7371 & 0.829940 & 3 & 5 & 11.7371 & 0.829940 \\
1 & 5 & 12.0950 & 0.855246 & 3 & 6 & 11.7371 & 0.829940 \\
1 & 6 & 12.2931 & 0.869251 & 4 & 5 & 11.7371 & 0.829940 \\
2 & 3 & 11.7371 & 0.829940 & 5 & 6 & 11.6040 & 0.820527 \\
2 & 5 & 11.7830 & 0.833184 & $*$ & $*$ & $*$ & $*$ \\
\hline
\end{tabular}

one considers larger and larger vertex sets, one realizes that there is a methodological infeasibility associated with the computational enumeration of all alternative topologies, for $\mathcal{R}$-sausages with more than six vertices. This enumerative procedure is an inductive dead end. There is often more than one alternative to the path topology, and one may not be able to rule out the emergence of one of these alternative topologies as optimal, at the next step.

A symbolic factorization appears difficult here even though a symbolic representation has not been difficult. A proof technique based on the "fold-up" of a semi-group property from two dimensions to three dimensions seems appropriate. This may be considered similar to the "inheritance of property" that is often encountered, in algebra, among vector spaces, fields, rings and groups, when an operator is "induced" on a subspace or subgroup. Given the non-scalar nature of the $\mathcal{R}$-sausage object, it turns out to be the fold-up of a semi-group property rather than a group property. This is understandable because the existence of an inverse element, which is a group axiom, is, for such a set element, meaningless.

Lemma 9. The binary composition defined in Lemma 6 for flat sausages, remains well defined on the set of all $\mathcal{R}$-sausages.

Proof. Though the $\mathcal{R}$-sausages are three dimensional, the Maxwell operation, alternately called a binary composition in the text, consisting of a split and optimization remains along the plane of the triangle. The steepest descent is along the negative of the left-hand gradient and a right-hand gradient:

$$
\nabla L(X, Y, Z)_{\delta h \rightarrow 0+}=\left[\partial L / \partial X_{\delta h \rightarrow 0+}, \partial L / \partial Y_{\delta h \rightarrow 0+}, \partial L / \partial Z_{\delta h \rightarrow 0+}\right]
$$

and

$$
\nabla L(X, Y, Z)_{\delta h \rightarrow 0-}=\left[\partial L / \partial X_{\delta h \rightarrow 0-}, \partial L / \partial Y_{\delta h \rightarrow 0-}, \partial L / \partial Z_{\delta h \rightarrow 0-}\right] .
$$

The gradients now have an additional $\partial L / \partial Z$ component. The number of minimizing directions also jumps up to three, but, just like in the case of flat sausages, the component of the gradient parallel to the axis of the composite $\mathcal{R}$-sausage sum to zero, and one of the components of the gradient is towards the axis of the composite element. The sum of the third components provide a turning force on the entire element, and does not affect the topology. 
Lemma 10. The set of all $\mathcal{R}$-sausages of finite length, in three dimensions, constitute an infinite abelian semi-group that is closed under the binary composition in Lemma 9. The topology exhibited by a typical element of this semi-group is called the path topology.

The set satisfies axioms 1-4 of Definition 4, under the binary composition in Lemma 9.

Theorem 7. The infinite $\mathcal{R}$-sausage in $E^{3}$ has the path topology.

Proof. By applying the binary composition defined in Lemma 9 a countably infinite number of times, the infinite $\mathcal{R}$-sausage is also a member of the semi-group of $\mathcal{R}$-sausages with the path topology.

\section{Summary}

In this article we have taken a close look at the flat sausage and the $\mathcal{R}$-sausage in two and three dimensions, respectively. The path topology, a full Steiner tree topology, was defined, and shown to be optimal for the flat sausage. An alphabet, where each of the letters is a characterization of a Steiner point in terms of its neighboring vertices, was presented. The path topology was shown to be equivalent to a converged sequence in this finite alphabet. This allows a classification of more complex Steiner topologies, as permutations and combinations of the path topology, for a specific traversal order. The path topology was then shown to be optimal also for the $\mathcal{R}$-sausage. This tightens our set of conjectures that the flat sausage and the $\mathcal{R}$-sausage have the best Steiner ratio in the plane and in three dimensional Euclidean space.

\section{References}

1. Cieslik, D. (2000). The Steiner Ratio. Kluwer, Dordrecht

2. Courant, R., and H. Robbins (1941). What is Mathematics? Oxford University Press, New York.

3. Coxeter, H. S. M. (1961). Introduction to Geometry. Wiley, New York, p. 412.

4. Du, D.-Z. (1991). Topological Network Design (J. M. Smith and P. Winter, eds.). Annals of Operations Research, Vol. 33, Baltzer, Basel, pp. 437-451.

5. Du, D.-Z. and F. K. Hwang (1992). A proof of the Gilbert-Pollak conjecture on the Steiner ratio. Algorithmica 7, 121-135.

6. Du, D.-Z. and W. Smith (1996). Disproofs of the Gilbert-Pollak conjecture on the Steiner ratio in three or more dimensions. J. Combin. Theory Ser. A 74, 115-130.

7. Du, D. Z., F. K. Hwang and J. F. Feng (1992). Steiner minimal trees on zig-zag lines, Trans. Amer. Math. Soc. 278(1), 149-156.

8. Garey, M. R., R. L. Graham and D. S. Johnson (1977). The complexity of computing Steiner minimal trees. SIAM J. Appl. Math. 32(4), 835-859.

9. Garey, M. R., and D. S. Johnson (1979). Computers and Intractability: A Guide to the Theory of NPCompleteness. Freeman, San Francisco, CA, pp. 208-209.

10. Gilbert, E. N., and H. O. Pollak (1968). Steiner minimal trees. SIAM J. Appl. Math. 16, 1-29. 
11. Rubinstein J. H., and D. A. Thomas (1991). A variational approach to the Steiner problem. Ann. Oper. Res. 33, 481-499.

12. Smith, J. M. (1980). Point set decomposition and suboptimal Steiner minimal trees. Presented at the TIMS-ORSA Meeting, Colorado Springs, CO.

13. Smith, W. D., and J. MacGregor Smith (1995). On the Steiner ratio in 3-space. J. Combin. Theory.

14. Smith, W. D. (1992). How to find Steiner minimal trees in Euclidean $d$-space. Algorithmica 7, 137-177.

Received November 29, 2001, and in revised form June 27, 2003. Online publication March 30, 2004. 